\title{
VASCULARIZATION OF THE CORNEA*
}

\author{
BY
}

\section{J. H. DOGGART}

London

THE subject of corneal vascularization becomes increasingly complicated as every year adds to the number and variability of factors known to promote the ingrowth of adventitious vessels. Some of the main queries that arise may be listed thus:

(i) What are the chief lesions associated with vascularization of the cornea ?

(ii) To what extent do the various modes of vascularization facilitate a differentia! diagnosis?

(iii) Is there a demonstrable factor common to the lesions concerned ?

(iv) What is the real significance of these adventitious vessels ?

(v) Does experimental work throw any light upon the problem ?

(vi) Is it possible to control the degree and duration of invasion by new vessels ?

\section{Corneal Lesions Liable to Vascularization}

Before considering those conditions in which adventitious vessels are the rule, it must be pointed out that intense vascularization may also arise for no obvious reason. Reference will later be made to such cases, which may reasonably be labelled "vascular keratitis of unknown origin". The following are the chief conditions in which new vessels invade the cornea.
(1) Phlyctenular disease
(2) Interstitial keratitis
(3) Sclerosing keratitis
(4) Disciform keratitis
(5) Trachoma
(6) Rosacea

(7) Various ulcers

(8) Mechanical irritation

(9) Chemical damage

(10) Intra-ocular disorganization

(11) Certain forms of nutritional disturbance

(12) Some of the dystrophies

(1) Phlyctenular Disease.-This condition commonly produces engorgement of the marginal loops before any characteristic yellowish dots become evident. These dots tend to encroach farther and farther on to the cornea, starting at the limbus. They are always accompanied by adventitious vessels but may fail to appear in recurrent attacks. In the cases exhibiting poor response to treatment we often find the clinical picture of fascicular keratitis, in which a leash of vessels embedded in cloudy corneal substance

\footnotetext{
* Received for publication January 20, 1951.
} 
tracks towards the axis. Although the original vascularization in phlyctenular disease is superficial, we may find deeper vessels in recurrent cases, especially when ulceration has been severe.

(2) Interstitial Keratitis.-Early in the active stage there may be one or more branches of superficial vessels, picturesquely called epaulettes, but the characteristic vascularization of this disease is deep. Until the œdematous infiltration has begun to subside, it may be impossible to secure a detailed view of these loops, which occupy the posterior one-third of the substantia propria.

(3) Sclerosing Keratitis.-This appears to be a tuberculous disease in many instances, and the new vessels, which are almost invariably present, may occupy any layer, but show a predilection for the posterior half of the substance.

(4) Disciform Keratitis.-This usually excites so much cloudy swelling and thickening of the axial region of the cornea that we may fail to follow the vessels across until clearing has begun. No case examined by me has ever shown complete clearance of the opacity, but improvement is usually sufficient to display the deep vessels, and incidentally the axial keratic precipitates, which at first had been concealed from view.

(5) Trachomatous Pannus.-This consists of a vascular infiltrate entering the superficial layers of the cornea from above, and seldom extending more than $1 \mathrm{~mm}$. beyond the transverse half-way line.

(6) Rosacea.-Conjunctivitis appears to pave the way for keratitis by keeping the vascular loops at the limbus in a state of intense engorgement. The corneal infiltrates occurring in this disease are always vascularized. At first only the superficial layers are invaded, but subsequent ulceration may be followed by the development of vessels at any level in the corneal substance. In a great majority of cases the lower half of the cornea is more widely implicated.

(7) Ulcers.-Those of any size nearly always display some vascularization in the course of healing, and indeed the absence of new vessels is part-evidence of devitalization when, for instance, the axial region of each cornea melts away as a consequence of keratomalacia. Other things being equal, recurrent ulceration is more likely to stimulate vessel-formation than is a single attack, and the dendritic variety is a good example of this generalization. Vascularized ulcers are also apt to arise as a complication in measles and small-pox, and occasionally in the bulbar form of spring catarrh (vernal conjunctivitis).

(8) Mechanical Irritation.-If it persists over a period of weeks or months this is a potent stimulus to the ingrowth of vessels from the conjunctiva. Numerous instances arise in the countries beset by trachoma, because the cicatricial stage of this disease tends to produce entropion. The consequent trichiasis leads to repeated 
epilation of the misdirected lashes which thus come to consist of short bristles. Trachoma is not, however, the only cause of entropion. Some cases are of traumatic origin, whereas some follow the vesico-bullous group of skin diseases, or prolonged blepharitis.

(9) Chemical Damage.--Since this is of almost infinite variety, it is seldom possible to give a reasonable prognosis when some unfamiliar substance has injured an eye. Certainly we cannot always judge the extent of permanent disability from the early clinical appearances. Sometimes the results of treatment surpass all expectation. In other cases an apparently trivial lesion must have been accompanied by some profound change in the anterior scleral and conjunctival vessels. Of this mustard-gas keratitis furnishes a striking example, for some of the victims who sustained relatively slight burns from this pernicious vesicant in 1917 and 1918 began to develop severe recurrent vascular keratitis after a lapse of anything up to 20 years; and evidence of deranged vascular supply was usually obvious in the form of multiple varicosities upon the conjunctiva.

Probably many another deleterious substance shows a time-lag before its full extent of destruction is unfolded, although we seldom find so long a latent period as obtains in burns by mustard-gas. Chromic acid is one of the more insidious agents of damage. In one case recently under my care, early progress was not maintained, and several months after the original injury the cornea became progressively opaque, new vessels advanced, and the intra-ocular pressure rose.

(10) Intra-Ocular Disorganization.-Whether it represents the endresult of absolute glaucoma, perforating injury, recurrent iridocyclitis, longstanding retinal detachment, or a combination of such disasters, this condition is often associated with the encroachment of superficial vessels upon the cornea.

(11) Nutritional Disturbance.-Vascularization of the cornea has been reported as one result of vitamin A deficiency, but presumably these were relatively mild cases. In the classical cases of keratomalacia due to lack of vitamin A, vessels were conspicuously absent, even under the provocation of secondarily infected ulcers, which would soon have promoted vascularization in the healthy subject.

Greater interest, however, has been aroused by the problem of adventitious corneal vessels in ariboflavinosis. A few years ago it became obvious that vitamin B2 deficiency was being diagnosed on insufficient evidence. Observers untrained in ophthalmology, and novices in the use of slit-lamp microscopy, were jumping to absurd conclusions on the strength of having witnessed slight engorgement of the peripheral or limbal arcade of vessels. . It will be remembered that normally a festoon of such collateral branches, aptly described by Mackenzie as an "anastomotic wreath", extends around the 
circumference of the limbus. Any sustained engorgement of the conjunctival vessels renders this limbal plexus more distinct, with the result that as soon as this sign was taken to be pathognomonic of ariboflavinosis innumerable mistakes arose.

To say this is not to deny that gross deficiency of riboflavin can provoke vascularization extending far beyond the peripheral festoon. Such a phenomenon has been observed by responsible investigators, and its aetiology confirmed by rapid shrinkage of the new-formed vessels in response to dosage with riboflavin. It seems clear, however, that genuine ariboflavinosis, if sufficiently advanced to provoke corneal vascularization, will also have given rise to unequivocal signs elsewhere, especially on the skin and mucous membranes. Therefore slit-lamp microscopy is not really a suitable method of routine diagnosis in this form of deficiency.

(12) Corneal Dystrophies. - In one member of this group (marginal degeneration of the cornea) new vessels are a constant feature. This disease of unknown origin affects men more often than women. It usually begins in the late teens or early twenties, and is commonly associated with recurrent attacks of conjunctivitis. Both eyes are affected, but manifestations in the second eye may be delayed for years. The earliest sign is a vascularized marginal opacity invading the corneal periphery from above, and one interesting feature is the clearly-marked whitish line whereby the affected area is bounded. This boundary relentlessly advances downward, always accompanied by superficial vessels, and sooner or later becomes a gutter, owing to slow destruction of the substantia propria. Meanwhile a fresh focus will probably have started from below, and ultimately the two will embrace the whole limbus. Before ever they have joined, however, the ectatic stage is likely to be evident in the upper, i.e., the original, seat of trouble. Progressive attenuation of the corneal substance, together with the thrust of the normal intra-ocular pressure, has led to spontaneous perforation in a number of recorded instances of this dystrophy.

Superficial marginal vascularization of the cornea may also occur in the later stages of familial keratitis or of Fuchs' epithelial dystrophy, but it is not one of the more important features of these conditions.

\section{New Vessels in Relation to Differential Diagnosis}

The mode of vascularization is an important point in differential diagnosis, so long as the facts are interpreted with discrimination and in relation to the other features of any particular case. We have already seen that superficial vessels are the rule in some conditions, and deep vessels in others. We have also noted the tendency of certain diseases to be located in one region of the cornea more than in another. Exceptions will from time to time arise. Therefore it 
is a mistake to draw up a rigid set of rules. In order to exploit the diagnostic significance of vascular variations we should assemble the rest of the clinical evidence, including the history, the marks of disease in other parts of the body, and the accessory ocular signs. Often a diagnosis will be obvious on general grounds, but sometimes observations upon the pattern of corneal vessels may be decisive.

Naturally it is difficult to make precise investigations upon a case of active keratitis showing much irritability and photophobia, but we have to remember that vascularization of the substantia propria leaves permanent traces. Long after the flush of new vessels has faded, close scrutiny of the cornea will disclose their shrivelled remnants, like delicate veining on the back of a leaf; and in many cases of systemic disease the true explanation of latent syphilis was first adumbrated by a ghostly corneal network. Such vestiges can usually be detected without recourse to the slit-lamp. Those who are not skilled in the use of a corneal loupe will find it easier to bring them into view by direct ophthalmoscopy with the aid of a high-powered convex lens in the sight-hole.

Diagnostic aid is also furnished in rosacea. Extensive corneal rosacea is usually accompanied by abundant signs upon the face, but cases do arise in which the skin betrays very little evidence of the disease. If we find vascularized scars of the characteristic wedge- or tongue-shape chiefly situated in the lower half of the cornea, then we may perhaps help to reach a diagnosis in time to apply prophylactic treatment.

Gardiner (1946) suggests that clues to the differential diagnosis of digestive disease can often be obtained from corneal vascularization, and that this sign is especially relevant as corroborative evidence of duodenal ulcer.

\section{Possible COMmon FACTORS}

At one time great emphasis was laid upon the role of mechanical irritation in the production of cancer, and many authorities postulated a latent or obscure source of irritation in cases exhibiting no crude agent. Notwithstanding instances in which no irritative factor.was apparent, it is true that irritation, whether mechanical, chemical, or other, is one of the more important factors.

In the same way, many of the aforementioned instances of vascular keratitis were clearly provoked by irritation, but some deeper influence must be at work, because the same irritant will produce new vessels in one case and not in another; and exuberant vesselformation often emerges without any such cause being manifest. Thus no common factor in thé production of new vessels in the cornea has yet been established, although irritant stimuli in great variety obviously play their part. Inflammation, oxygen-want, and changes 
in the hydrogen-ion concentration are among the other possible contributory influences.

\section{Significance of Corneal Vascularization}

Much knowledge about the cornea had already been accumulated when Bowman (1849), referring to pannus, wrote:

unless the vessels had been developed, the disease process would long ago have terminated by the total destruction of the tissue.

It is clear that invasion of the cornea by new vessels is, on the whole, a beneficent process, a means of scavenging the substantia propria; this clinical impression is fortified by animal experiments.

The main significance of vascularization may therefore be described as reparative, but we have to recognize that, just as epithelial proliferation may pass beyond the bounds of repair until it creates havoc, so may a leash of adventitious vessels, originally budding forth to heal, slip into devastating proliferation. Keratitis involving the whole surface in dense vascularized opacity may be set in train by a slight abrasion, or may even arise spontaneously, in those who are cursed with the proclivity to sprout blood vessels. From the clinical standpoint the two most important facts are these:

(1) Invasion of the cornea by new vessels is an integral part of the healing process in many different lesions.

(2) Disproportionate and mysteriously progressive vascularization is unfavourable.

\section{EXPERIMENTAL WORK}

Owing to its transparency, superficial situation, and small size, the cornea has been a convenient vehicle for experimental work upon the development of new vessels. The effect of various irritant substances has been investigated by numerous observers. Campbell and Michaelson (1949) came to the conclusion that the immediate stimulus for new vessel-formation was some factor diffusing from the lesion itself. They used a platinum-wire cautery to produce standardized burns in rabbits' corneae, and noticed that a burn inflicted beyond a certain critical distance from the limbus failed to produce vascularization. Within the critical distance a burn resulted in a vascularized triangle with its base at the limbus. Indian ink injected into the carotid served to map-out such areas.

Vascularization of animals' corneae from riboflavin deficiency has been the subject of a vast amount of experimental work, but it has been found that diet defective in various amino-acids can also incite vessel-formation. . Modern preoccupation with riboflavin is exemplified by the fact that this substance has been widely used in the treatment of phlyctenular disease and rosacea-fertile sources of adventitious vessels.

Cogan (1949) believes that the deciding factor is a reduction in the compactness of the tissue stroma. He points out that oedema 
supplies the haze so often associated with the ingrowth of new vessels, and suggests that diminution in the swelling of the corneal substance in a case of interstitial keratitis directly follows the vascular invasion. Cogan also worked on the experimental production of bullous keratopathy by hypertonic-saline injections into the anterior chamber, and found that this condition took a more severe form in the normal cornea than in one already vascularized.

The work of Meyer (1940-48) suggests that the transparency, water-content regulation, and freedom from blood vessels of the normal cornea depend upon the presence of a polysaccharide, probably represented in the human subject by hyaluronosulphate. It will be remembered that the related substance, hyaluronic acid, which is present in synovial fluid, appears to act as a shock-absorber. Hyaluronosulphate is distributed through the interfibrillar spaces of the cornea's collagenous network, and its discovery presents tremendous possibilities.

It seems fair to summarize the position by saying that experimental work has already illuminated the problem of corneal vascularization. although much more knowledge is required. Perhaps one warning should be given regarding the interpretation of experiments. Much of the work has been done on rabbits, and it so happens that these animals can develop new corneal vessels with astonishing facility. Therefore a similar response to any particular agent of vascularization must not be assumed as inevitable when human beings or other animals are likewise exposed.

\section{Control of Vascularization in Clinical Practice}

The direct control of vascularization is difficult, and would in most cases be undesirable. Thus, for instance, the obvious purpose of the invading vessels in both disciform and interstitial keratitis is to clear away the oedamatous infiltrate and cellular debris with which the interlamellar spaces are clogged; so that artificial strangulation of the adventitious twigs could only prolong or aggravate the essential lesion, as Bowman long ago perceived. Indire't control of new vessels is another matter. As a rule we can shrivel up such branches by rendering them unnecessary-that is to say, by eliminating the factors which provoked their formation. Genuine cases of ariboflavinosis displaying extensive corneal vascularization will soon respond to dosage with riboflavin, and there are on record many authentic instances in which rapid withering of vessels was seen to follow that treatment. To take another example: suppose that vessels were beginning to invade a neglected corneal ulcer, and that treatment were then initiated. Perhaps the conjunctival sac and the surface of the ulcer would be flushed clean, suitable chemotherapy administered, and some form 
of cauterization applied. Then, in a large proportion of cases, the ingrowth of new vessels would stop, simply because healing had now been rendered possible without their aid.

Reference has already been made to those cases of vascular keratitis in which the vessels themselves, together with their cloudy pathways, menace the welfare of the cornea and serve no useful purpose, having started to multiply in the absence of any ordinary provocation. Many of the older clinicians used to perform the operation of peritomy with the object of checking the process of vascularization in such cases, but were seldom successful. Fresh anastomotic networks were soon flung out and it is clear that, when the propensity to form new vessels is really strong, minor surgery will not avail, and the only operative procedure likely to stop it would be too drastic to be feasible-something that would interfere with the entire circulatory system of the eyeball.

Cogan's experiments on artificially produced bullous keratopathy led him to wonder whether such a condition encountered in practice might derive benefit from the setting up of interstitial vascularization. It will be interesting to hear what measure of success he achieves when these investigations are completed. It is plainly more difficult to promote interstitial than superficial vascularization by artificial means. Moreover, the chances of permanent damage ensuing from the ingrowth of deep vessels are by no means negligible. Nor will it be easy to find cases of bullous keratitis likely to gain better sight from amelioration of that condition, since it is so frequently associated with grave disease of the inner eye.

\section{CONCLUSIONS}

Clinical ophthalmologists can derive a mass of helpful information from observing the advance and shrinkage of new vessels invading the cornea. Their situation and depth, and the associated signs of diseases elsewhere in the body-including the fellow-eye in cases apparently unilateral-will offer many clues to diagnosis, treatment, and prognosis. It is to be hoped that clinical observers working in close accord with experimentalists will ultimately discover how to check the development of vessels which fulfil no useful purpose, or persist long after their work of repair has come to an end.

REFERENCES

Bowman, W. (1849). "Lectures on the Parts concerned in Operations on the Eye", p. 32. Longmans, London.

Campbell, F. W., and Michaelson, I. C. (1949). British Journal of Ophthalmology, 33, 248.

Cogan, D. G. (1949). Arch. Ophthal., Chicago, 41, 406.

(1949). Amer. J. Ophthal., 32, 625

Gardiner, P. A. (1946). British Journal of Ophthalmology, 30, 225, 581.

MeYer, K. (1947). Physiol. Rev., 27, 335.

(1948). in A. Sorsby "Modern Trends in Ophthalmology", vol. 2, p. 71. Butterworth, London.

and Chaffee, E. (1940). Amer. J. Ophthal.. 23, 1320. 